\title{
Propionibacterium granulosum
}

National Cancer Institute

\section{Source}

National Cancer Institute. Propionibacterium granulosum. NCI Thesaurus. Code C86682.

A species of anaerobic, Gram-positive, rod shaped bacteria assigned to the phylum Actinobacteria. This species is non-spore forming, catalase positive, indole negative, does not hydrolyze esculin, starch, gelatin, or casein, and does not reduce nitrate. P. granulosum has been isolated from the skin and gastrointestinal tract, and may contribute to the etiology of acne vulgaris. 